\title{
SUBSTRUCTURE IN CDM HALOS AND THE HEATING OF STELLAR DISKS
}

\author{
Julio F. Navarro \\ Department of Physics and Astronomy, \\ University of Victoria, \\ Victoria, BC, V8P 1A1, Canada
}

\begin{abstract}
Numerical simulations have revealed the presence of long-lived substructure in Cold Dark Matter (CDM) halos. These surviving cores of past merger and accretion events vastly outnumber the known satellites of the Milky Way. This finding has prompted suggestions that substructure in cold dark matter (CDM) halos may be incompatible with observation and in conflict with the presence of thin, dynamically fragile stellar disks. N-body simulations of a disk/bulge/halo model of the Milky Way that includes several hundred dark matter satellites with masses, densities and orbits derived from high-resolution cosmological CDM simulations indicate that substructure plays only a minor dynamical role in the heating of the disk. This is because the orbits of satellites seldom take them near the disk, where their tidal effects are greatest. We conclude that substructure might not preclude virialized CDM halos from being acceptable hosts of thin stellar disks like that of the Milky Way.
\end{abstract}

\section{Introduction}

One recent highlight of cosmological N-body simulations has been the discovery that during the merger and accretion events that characterize the assembly of dark matter halos the central regions of accreted halos may survive for several orbital times as dynamically distinct, self-bound entities in the parent halo (Klypin et al 1999, Moore et al 1999, hereafter K99 and M99, respectively). The population of surviving halo cores, or "subhalos", typically contributes less than $\sim 10 \%$ of the total mass of the system, with the bulk of the mass in a smooth monolithic structure, as envisioned in the pioneering analytical work of White \& Rees (1978). Despite the small fraction of the total mass they make up, at any given time a large number of subhalos are expected within the virialized region of a cold dark matter halo. For example, M99 find that up to 500 satellites with circular velocities exceeding $\sim 10 \mathrm{~km} / \mathrm{s}$ may have survived within $\sim 300 \mathrm{kpc}$ from the center of the Galaxy. Comparing this with the dozen or so known Milky Way satellites of comparable velocity dispersion implies that most subhalos must have failed to form a significant number of stars.

Even if luminous galaxies fail to "turn on" in most subhalos a potential difficulty has been cited by M99: the fluctuating gravitational potential induced by the clumpy structure of the halo may act to heat and thicken fragile 
stellar disks beyond observational constraints. I present here the results of an attempt to quantify the effects of substructure on the dynamical evolution of thin stellar disks embedded in dark matter halos.

\section{Substructure in CDM halos}

The mass function of substructure halos, their internal structure, and the parameters of their orbits are the main properties of the subhalo population that determine the tidal effects of substructure on stellar disks. The short-dashed curves in Figure 1 (left panel) show the subhalo velocity function corresponding to galaxy-sized dark matter halos formed in the $\Omega=1 \mathrm{CDM}$ and in the $\Lambda \mathrm{CDM}$ cosmogonies. Circular velocity (instead of mass) is used to characterize subhalos because of its weaker dependence on the exact way in which substructure is identified. Still, circular velocities do change as a function of radius from the center of a subhalo, and there is no unique way of defining subhalo circular velocities. Figure 1 reports results for $V_{\text {peak }}$ and $V_{\text {outer }}$, which correspond to the maximum circular speed within the subhalo, and to its value at the outermost bound radius, respectively. Scaled to the virial velocity of the halo, the substructure velocity function is roughly independent of the mass of the parent halo (M99) and of the cosmological parameters (K99). The results of K99 are shown by the dot-dashed line in Figure 1 (left) and are in reasonable agreement with our determination.

How does the subhalo velocity function compare with the observed number of satellites in the vicinity of the Milky Way? The solid squares in Figure 1 (joined by a solid line) illustrate the cumulative number of known Milky Way satellites as a function of the circular velocity of their halos, as plotted by M99. Here, circular velocities are derived for the halos of dwarf spheroidals assuming that stars in these systems are on isotropic orbits in isothermal potentials. This is a plausible, but nevertheless questionable, assumption. Dark halos differ significantly from simple isothermal potentials, and numerical simulations indicate that circular velocities decrease substantially near the center. If stars populate the innermost regions of subhalos their velocity dispersions may substantially underestimate the subhalo peak circular velocities. This has been discussed by White (2000), who finds, using the mass model proposed by Navarro, Frenk \& White (1997, NFW), that dwarf spheroidals may plausibly inhabit potential wells with circular velocities up to a factor of 3 times larger than inferred under the isothermal assumption. Such correction may reconcile, at the high mass end, the Milky Way satellite velocity function with the subhalo function, as shown by the dotted line in Figure 1. Thus, the possibility

remains that the number of massive satellites expected in the CDM scenario 

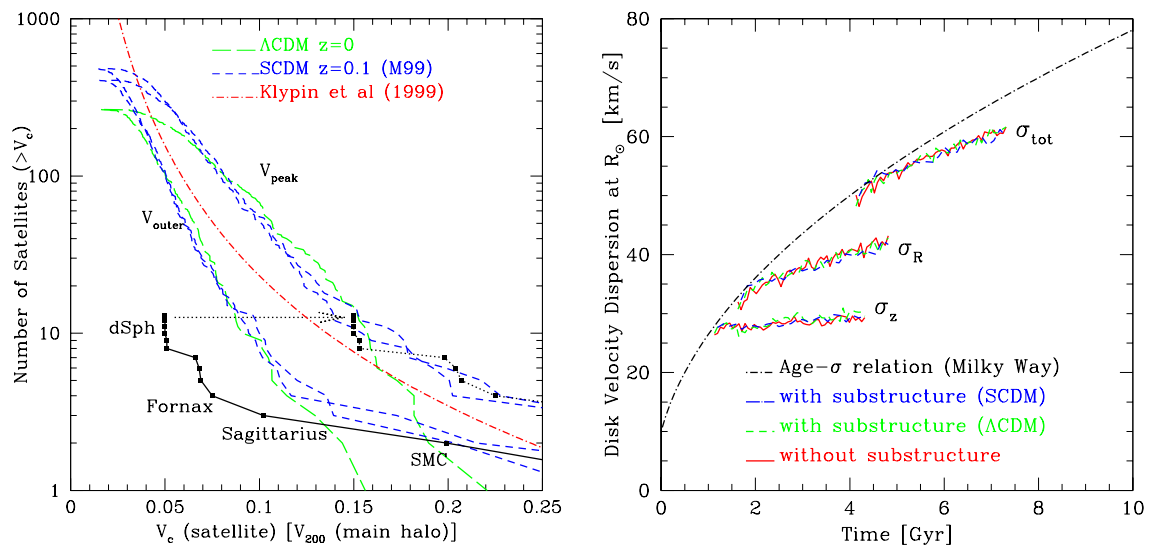

Figure 1: Left: Cumulative circular velocity function of substructure halos. Velocities are normalized to the circular velocity of the parent halo measured at the virial radius. Only subhalos within the virial radius of the main halo are included. Right: Stellar disk velocity dispersion as a function of time in our Milky Way model compared with the age-velocity dispersion relation in the solar neighborhood, as compiled recently by Fuchs et al (2000, dot-dashed curve).

might not be in gross conflict with observation.

On the other hand, this does not erase the large number of low mass "dark" satellites that should inhabit the main halo. Can thin stellar disks survive unscathed in this clumpy environment? The tidal heating rate by substructure can be shown to scale as $d E / d t \propto \int n\left(m_{s}\right) m_{s}^{2} d m_{s}$, where $m_{s}$ is the subhalo mass (White 2000). Since, according to numerical simulations, $n\left(m_{s}\right) \propto m_{s}^{-1.8}$ (Ghigna et al 2000, Springel et al 2000), tidal heating by substructure is dominated by the few most massive subhalos. One consequence of this result is that stochastic effects are expected to dominate the heating rate and a statistically significant sample of systems should be simulated in order to obtain conclusive results. The results reported here (which are based on a couple of realizations) should then be viewed as preliminary until confirmed by further studies.

\section{The Evolution of Stellar Disks in a $\Lambda$ CDM halo}

In order to assess the dynamical influence of subhalos on stellar disks we have carried out a series of numerical simulations that follow the evolution of a disk of particles within a dark matter halo with substructure similar to that of the 
halos shown in Figure 1 (left). We choose parameters so that the model reproduces many of the observational characteristics of the Milky Way galaxy, as in Velázquez \& White (1999). The galaxy model is first evolved without substructure halos in an attempt to assess the deviations from equilibrium induced by noise in the particle distribution. N-body disks are notoriously unstable, and substantial numbers of particles are needed to ensure stability over several rotation periods. The simulations we report here use 40,000 particles in the disk, 13,333 in the bulge, and $2.2 \times 10^{6}$ in the halo, all of equal mass, $m_{p}=1.4 \times 10^{6} M_{\odot}$. Within this system we insert substructure halos, using a procedure which ensures that the subhalos have approximately the same masses, densities and orbits as those in the CDM halos.

We evolve the disk model first for about 3.5 Gyrs without including substructure in order to quantify the heating rate due to noise in the particle distribution. The $(R, z, \phi)$ velocity dispersions of disk particles at the solar circle (i.e., $8.5 \mathrm{kpc}$ from the center) grow from $(31,27,26) \mathrm{km} \mathrm{s}^{-1}$ to $(43,30$, 33) $\mathrm{km} \mathrm{s}^{-1}$ over the same period (see solid lines in Figure 4). Quantifying this heating rate by the usual expression $\sigma_{\text {tot }}^{2}=\sigma_{0}^{2}+D t$, we find $D \sim 200$ $\mathrm{km}^{2} \mathrm{~s}^{-2} \mathrm{Gyr}^{-1}$, about a factor of two less than inferred for stars in the solar neighborhood from the age-velocity dispersion (Fuchs et al 2000).

The two-body heating rate in our equilibrium stellar disk model is thus low compared with the actual heating experienced by stars in the disk of the Galaxy. This implies that our disk model is stable enough to verify numerically whether substructure in the halo leads to heating rates inconsistent with observational constraints. This is shown by the dashed lines in Figure 4, which show the evolution of the disk velocity dispersion when the substructure halos are added to the system. Clearly, the heating rate is approximately the same with and without substructure, a result that may be traced to the fact that there are no satellites more massive than $10^{11} M_{\odot}$ and that their orbits seldom take them near the disk. If substructure in the halos we consider is representative of galaxy-sized CDM halos (and we have no reason to suspect it is not), this would imply that tidal heating rates of thin stellar disks by substructure halos may be consistent with the observational evidence.

\section{Discussion}

These results suggest that concerns regarding excessive tidal heating of thin stellar disks by substructure in CDM halos may be less serious than previously thought. We conclude that the substructure observed in virialized CDM halos is not clearly inconsistent with the existence of thin stellar disks such as that of the Milky Way. These conclusions are subject to a number of caveats. The 
most obvious one is that our study explores only two numerical realizations of a disk galaxy within clumpy dark matter halos, and it is always hazardous to extrapolate from such a small number of cases. Our study does show, however, that it is possible at least in some cases to maintain a stellar disk in spite of substructure. A second caveat is that we have explored a model motivated by the present-day structure of the Milky Way and by the $z=0$ substructure of a CDM halo. Models that take into account the ongoing formation of the disk and a more realistic treatment of the evolution of substructure are clearly desirable in order to refine the conclusions presented here.

Finally, as discussed by Navarro \& Steinmetz (2000), it is quite difficult to account simultaneously for the masses, luminosities, rotation speeds, and angular momenta of galaxy disks in cosmogonies such as CDM, where much of the mass of a virialized halo tends to be assembled through a sequence of mergers. Until these issues are fully resolved it would be premature to extend a clean bill of health to the CDM paradigm regarding the formation and evolution of spiral galaxies like our own Milky Way.

\section{Acknowledgments}

I thank my collaborators, Andreea Font, Joachim Stadel and Tom Quinn for allowing me to present the results of our collaboration. This research has been supported by CIAR, CFI, NSERC and the Alfred P. Sloan Foundation.

\section{References}

1. Fuchs, B. et al. 2000, in "Dynamics of Star Clusters and the Milky Way", ASP Conference Series, eds. Deiters et al.

2. Ghigna, S. et al. 2000, ApJ, 544, 616

3. Klypin, A. et al, 1999, ApJ, 516, 530

4. Moore, B. et al, 1999, ApJl, 524, L19 (M99)

5. Navarro, J. F., Frenk, C. S. \& White, S. D. M. 1997, ApJ, 490, 493

6. Navarro, J. F. \& Steinmetz, M. 2000, ApJ, 538, 477.

7. Springel, V., White, S.D.M., Tormen, G., Kauffmann, G. 2000, MNRAS, submitted (astro-ph/0012055)

8. Velázquez, H. \& White, S. D. M. 1999, MNRAS, 304, 254

9. White, S. D. M. \& Rees, M. J. 1978, MNRAS, 183, 341

10. White, S.D.M. 2000, "ITP Conference on Galaxy Formation and Evolution", http://online.itp.ucsb.edu/online/galaxy_c00/white/ 